\title{
Nematic-isotropic interfaces under shear: A molecular-dynamics simulation
}

\author{
Guido Germano ${ }^{\text {a) }}$ \\ Fakultät für Physik, Universität Bielefeld, Universitätsstraße 25, D-33615 Bielefeld, Germany \\ and Fachbereich Chemie, Philipps-Universität Marburg, D-35032 Marburg, Germany \\ Friederike Schmid ${ }^{\text {b) }}$ \\ Fakultät für Physik, Universität Bielefeld, Universitätsstraße 25, D-33615 Bielefeld, Germany
}

(Received 28 April 2005; accepted 6 October 2005; published online 6 December 2005)

\begin{abstract}
We present a large-scale molecular-dynamics study of nematic-paranematic interfaces under shear. We use a model of soft repulsive ellipsoidal particles with well-known equilibrium properties, and consider interfaces which are oriented normal to the direction of the shear gradient (common stress case). The director at the interface is oriented parallel to the interface (planar). A fixed average shear rate is imposed with moving periodic boundary conditions, and the heat is dissipated with a profile-unbiased thermostat. First, we study the properties of the interface at one particular shear rate in detail. The local interfacial profiles and the capillary wave fluctuations of the interfaces are calculated and compared with those of the corresponding equilibrium interface. Under shear, the interfacial width broadens and the capillary wave amplitudes at large wavelengths increase. The strain is distributed inhomogeneously in the system (shear banding), the local shear rate in the nematic region being distinctly higher than in the paranematic region. Surprisingly, we also observe (symmetry-breaking) flow in the vorticity direction, with opposite direction in the nematic and the paranematic state. Finally, we investigate the stability of the interface for other shear rates and construct a nonequilibrium phase diagram. (C) 2005 American Institute of Physics.
\end{abstract} [DOI: 10.1063/1.2131065]

\section{INTRODUCTION}

Complex fluids often show peculiar behavior under shear. ${ }^{1}$ Particularly remarkable phenomena are observed in fluids with anisotropic components, which can exhibit a local or global orientational order. ${ }^{2,3}$ Shear flow tends to orient the materials, which usually reduces the shear viscosity (shear thinning), ${ }^{4-7}$ but can also have the opposite effect. ${ }^{5,8}$ In the vicinity of equilibrium phase transitions, shear flow may shift the boundaries and enforce ordering transitions. ${ }^{9-14}$ It may even induce the formation of new structures that are inhomogeneous in space or time, such as multilamellar vesicle phases in surfactant systems ${ }^{15,16}$ or tumbling states in liquid-crystalline polymers. ${ }^{17-21}$

In recent years special attention has been given to situations where phases "coexist" under shear. The interest was originally raised by the "spurt effect" in polymer melts, i.e., the observation that the flow rate in a pipe may change discontinuously as a function of the applied pressure difference. $^{22}$ This was attributed to a nonequilibrium phase transition from an entangled polymer phase with high viscosity to a highly aligned phase with low viscosity. ${ }^{4,23,24}$ The instability results from a nonmonotonicity in the (hypothetical) homogeneous stress-strain flow curve: In a homogeneous system, the stress would first grow with a growing strain rate and then decrease beyond a certain critical value. Since such a flow curve is mechanically unstable, the system becomes inhomogeneous. The stress plateau in the stress-

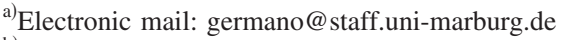

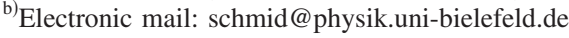

strain flow curve was interpreted as the signature of a region where phases with different shear rates coexist. The separation of a fluid into two states with distinctly different shear rates is commonly called shear banding.

Meanwhile, shear banding has been observed and even directly visualized in various systems, most notably wormlike micelles ${ }^{25-29}$ and rodlike viruses. ${ }^{21}$ More generally, two qualitatively different types of phase separation are possible in shear flow. ${ }^{30}$ These are illustrated in Fig. 1 for the Couette geometry. In the shear-banding case described above, the fluid separates in the direction of the flow gradient, the phases are subject to the same stress, and the shear rates differ (common stress case). Alternatively, the fluid can also phase separate in the direction perpendicular to both flow and flow gradient, the vorticity direction. The two phases then share the same shear profile, but the stress differs (common strain case).

The phase-separation mechanism described above is driven by mechanical (hydrodynamical) forces. However, phase separation and shear banding can also occur as a result of a thermodynamic phase transition-either a real transition that will also occur in the absence of shear flow, or a transition between phases that are metastable ("hidden") in equilibrium. Shear banding is often associated with ordering phenomena and sometimes occurs in the vicinity of a thermodynamic phase boundary. Thermodynamic forces then contribute to the nonmonotonic stress-strain relationship which gives rise to the mechanical instability. Thus the mechanical and the thermodynamical phase separation cannot always be separated unambiguously. ${ }^{31}$ In fact, theoretical 


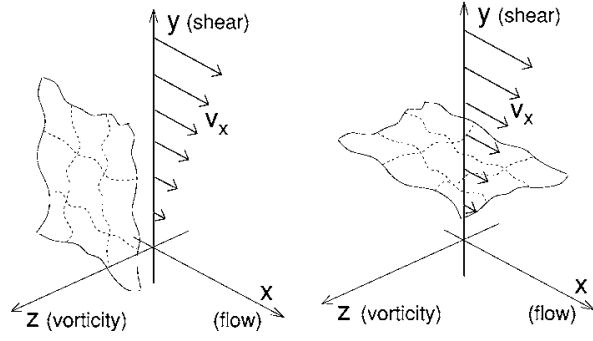

FIG. 1. Possible orientations of an interface: common strain (left) and common stress (right) geometries. Also indicated is the reference system used throughout this paper. In this paper, only the common stress case is investigated.

treatments of shear banding are often based on free-energy expressions for phase-separating systems. ${ }^{32-35}$

One should, however, note an important difference between mechanically and thermodynamically driven phase separations. ${ }^{36}$ The signature of mechanically driven phase coexistence is the existence of a plateau in the stress-strain flow curve. In the common stress case, the plateau is horizontal, while in the common shear case, it is vertical. ${ }^{30}$ If one increases the average shear rate/average stress in the plateau region, i.e., the coexistence region, the width of the coexisting bands readjusts, but the local stress and the shear rate in each phase remain the same. For thermodynamically driven phase separation, however, a free readjustment is only possible if solely nonconserved quantities are involved in the phase transition. If the two coexisting phases differ with respect to the density of a conserved quantity (e.g., a concentration), the overall average of this density imposes an additional constraint. As a result, both the bandwidth and the coexisting phases must be readjusted, and the "plateau" acquires a finite slope. ${ }^{34,35,37}$

This raises an important question: How does the system determine the location of the plateau and select the coexisting states? According to Olmsted and co-workers, the selection criterion is the requirement that a steady interface between stable coexisting homogeneous states exists. ${ }^{32,34,35}$ Hence the understanding of the interface provides the key to the understanding of the properties of the material under shear.

In the present work, we present a molecular-dynamics simulation of a nematic/isotropic (more precisely, paranematic) interface under shear, in a situation where the interface is already present in the absence of shear. The phase separation is thus driven by thermodynamic forces. The equilibrium properties of isotropic/nematic interfaces have been studied by computer simulations in a number of systems. ${ }^{38-44}$ Our study is designed such that we can compare the equilibrium and the nonequilibrium structure of the interface in as much detail as possible. Therefore, we choose a model of soft ellipsoids, which we have investigated extensively in the past, ${ }^{41,42,45-48}$ and used for large-scale simulations of equilibrium interfaces. ${ }^{41,42}$ These interfaces are now subjected to shear. Previous particle-based simulations of liquid crystals under shear have focused on the structure and the response functions in homogeneous phases. ${ }^{6,49-58}$ To our best knowledge, our simulation is the first particle-based nonequilibrium simulation of a liquid-crystal interface under flow.
Our paper is organized as follows: In Sec. II, we introduce the model and discuss the simulation method. The results are presented in Sec. III. We first discuss the stability of the interface with increasing shear rate, and then consider in detail the interfacial structures at one particular shear rate. We summarize and conclude in Sec. IV.

\section{MODEL AND METHOD}

We consider a system of soft repulsive ellipsoids with length-to-width ratio $\sigma_{l} / \sigma_{0}=15$. Every ellipsoid is characterized by the position of the center of mass $\mathbf{r}$ and a unit vector $\hat{\mathbf{u}}$ pointing in the direction of the long axis. The interaction potential between two ellipsoids $i$ and $j$ separated by the center-center vector $\mathbf{r}_{i j}$ is given by

$$
U= \begin{cases}4 \epsilon_{0}\left(s_{i j}^{-12}-s_{i j}^{-6}\right)+\epsilon_{0}, & s_{i j}<2^{1 / 6} \\ 0, & \text { otherwise, }\end{cases}
$$

where $s_{i j}=\left(r_{i j}-\sigma\left(\hat{\mathbf{r}}_{i j}, \hat{\mathbf{u}}_{i}, \hat{\mathbf{u}}_{j}\right)+\sigma_{0}\right) / \sigma_{0}$ is a scaled and shifted distance and $\sigma$ approximates the contact distance of two ellipsoids, ${ }^{59}$

$$
\begin{aligned}
& \sigma\left(\hat{\mathbf{r}}_{i j}, \hat{\mathbf{u}}_{i}, \hat{\mathbf{u}}_{j}\right)=\sigma_{0}\left\{1-\frac{\chi}{2}\left[\frac{\left(\hat{\mathbf{u}}_{i} \cdot \hat{\mathbf{r}}_{i j}+\hat{\mathbf{u}}_{j} \cdot \hat{\mathbf{r}}_{i j}\right)^{2}}{1+\chi \hat{\mathbf{u}}_{i} \cdot \hat{\mathbf{u}}_{j}}\right.\right. \\
& \left.\left.+\frac{\left(\hat{\mathbf{u}}_{i} \cdot \hat{\mathbf{r}}_{i j}-\hat{\mathbf{u}}_{j} \cdot \hat{\mathbf{r}}_{i j}\right)^{2}}{1-\chi \hat{\mathbf{u}}_{i} \cdot \hat{\mathbf{u}}_{j}}\right]\right\}^{-1 / 2}, \\
& \text { with } \chi=\frac{\left(\sigma_{l} / \sigma_{0}\right)^{2}-1}{\left(\sigma_{l} / \sigma_{0}\right)^{2}+1} \text {. }
\end{aligned}
$$

The particles have the mass $m$ and the moment of inertia $I=50 m \sigma_{0}^{2}$. For convenience, we choose the units such that $\sigma_{0}=\epsilon_{0}=m=1$. This defines the time unit $\tau=\sigma_{0} \sqrt{m / \epsilon_{0}}$. The temperature was $k_{B} T=\epsilon_{0}$.

In our previous work, ${ }^{42}$ we have performed an equilibrium molecular-dynamics simulation of a system with 115200 particles in an elongated box with side ratios (1:2:1) and periodic boundary conditions, at a density in the coexistence region, $\rho=0.017 / \sigma_{0}^{3}$. The system thus contained an isotropic and a nematic slab, separated by two interfaces. The director in the nematic phase was found to align parallel to the interface (planar). Within that plane, the direction of the director is of course arbitrary, but the initial equilibration stage was conducted such that it pointed roughly in the direction of one box side.

The final configurations from these simulations were used as starting configurations in the present work. Shear flow was enforced by applying Lees-Edwards boundary conditions, ${ }^{60-62}$ i.e., the periodic boundaries in one direction move at constant speed. This minimizes surface effects, but at the expense of constantly pumping energy into the system. In order to dissipate the latter, we have coupled the molecular velocities to a Nosé-Hoover thermostat, ${ }^{63}$ taking care that it acts only on the thermal fluctuations of the velocities, i.e., the velocities minus the streaming velocity of the fluid. The streaming velocity profile was determined from the simulation. In a nonuniform system like ours, using such a profileunbiased thermostat ${ }^{62}$ is obviously important. However, we have noted in preliminary studies that we get practically the 
same results with a profile-biased thermostat, where the actual velocity profile is approximated by a constant gradient profile. Hence the results do not seem to depend sensitively on the details of the thermostat.

In the present paper, we discuss the interfaces in the common stress geometry (Fig. 1, left). Two situations were considered: In the first, termed "flow-aligned" case, the initial configuration was turned such that the director in the nematic phase points in the direction of the flow, i.e., the $x$ direction in Fig. 1. In the second, "log-rolling" case, the initial configuration was turned such that the director points in the direction of the vorticity, i.e., the $z$ direction. We have first examined the stability of the interface in the flowaligned case for global strain rates up to $\dot{\gamma}=\Delta v_{x} / L_{y}=0.1 / \tau$. $\left(\Delta v_{x}\right.$ is the streaming velocity difference between both sides of the box, as imposed by the Lees-Edwards boundary conditions, and $L_{y}$ the box length in the $y$ direction.) To this end, we have carried out a series of simulations of smaller systems with 7200 particles in a box with side ratios $(1: 8: 1)$. Then we have focused on the strain rate $\dot{\gamma}=0.001 / \tau$, and performed extensive simulations of interfaces with initial flow-aligned and log-rolling setups. In both cases the system was first "equilibrated" over $2 \times 10^{6}$ molecular-dynamics (MD) steps, and data were then collected over the next $2 \times 10^{6}$ MD steps, where one step corresponds to 0.0015 time units $\tau$. In the flow-aligned case, the system remained flow aligned throughout the simulation. In the log-rolling case, two things happened during the initial "equilibration" time: The director of the nematic phase turned very slowly into the flow direction, and the density of the nematic phase decreased slightly (implying that the thickness of the paranematic slab shrank). After the initial equilibration time, the system had reached a state where half of the nematic slab was oriented at $45^{\circ}$ to the flow, and the director of the other half was still perpendicular to the flow (see Fig. 2). This state did not change anymore over the next $2 \times 10^{6} \mathrm{MD}$ steps, in which the data were collected. Thus we could analyze the properties of this long-lived, yet presumably metastable state and compare them with the properties of the stable flowaligned interface.

Since we use periodic boundary conditions, the interfaces can move freely through the system. Moreover, they exhibit capillary wave fluctuations, ${ }^{42,64-66}$ which broaden the apparent profiles and make their width dependent on the lateral system size. In order to eliminate these effects and determine local interfacial profiles, we adopt a block analysis technique introduced in our earlier studies of equilibrium interfaces: ${ }^{42}$ We split the system of size $L \times L_{y} \times L$ into columns of block size $B \times L_{y} \times B$. In the $y$ direction, the columns are further divided into 100 bins. Then we compute the local order tensor ${ }^{2}$ in each bin,

$$
\mathbf{Q}=\frac{1}{N} \sum_{i=1}^{N}\left(\frac{3}{2} \hat{\mathbf{u}}_{i} \otimes \hat{\mathbf{u}}_{i}-\frac{1}{2} \mathbf{I}\right),
$$

where $\mathbf{I}$ is the $3 \times 3$ unity matrix. The nematic order parameter $S(y)$ in the bin centered at $y$ is the highest eigenvalue of $\mathbf{Q}$ in that bin. From the profile $S(y)$, we determine the local positions $y_{N I}$ and $y_{I N}$ of the two interfaces in the column a)

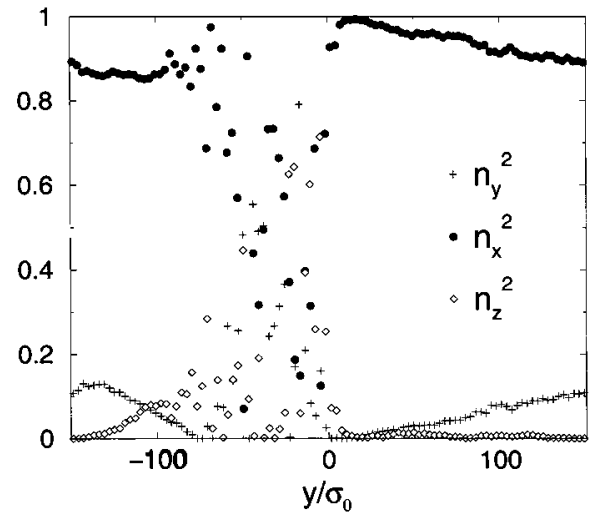

b)

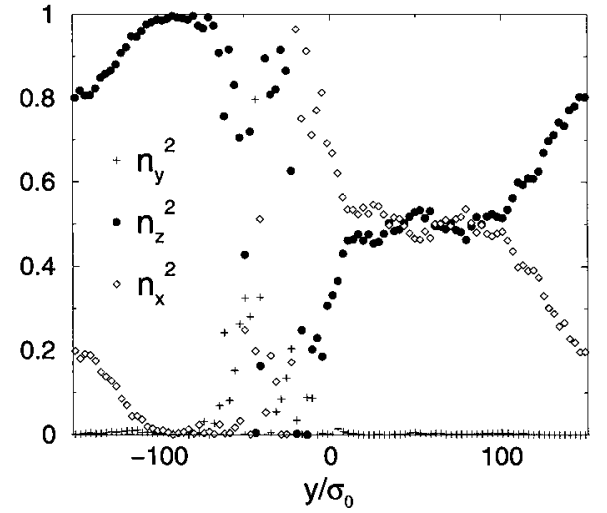

FIG. 2. Director orientation profiles in the final configuration of the interface set up in the (a) flow-aligned and (b) log-rolling geometries, with $N=115200$ particles. In the isotropic slab, which is situated between $y=-80 \sigma_{0}$ and $y=0$, the direction of the director is not well defined. The symbols show the squared component $n_{\alpha}^{2}$ of the director in different directions: the shear gradient direction (plus, $\alpha=y$ ), the director direction in the initial setup (closed circles), and the remaining direction (open diamonds). After the initial "equilibration" time, these profiles stay roughly constant throughout the simulation.

under consideration, following the procedure described in Ref. 42. Then we calculate the profiles for all quantities of interest and shift them by the amount $y_{N I}$ or $y_{I N}$, respectively. This allows us to perform averages over local profiles. Here, profiles from $N I$ interfaces are mirrored at $y_{N I}$ such that the nematic slab is always on the right side. The two interfaces from the log-rolling setup are discussed separately, since they were different (cf. Fig. 2). The left interface, where the director on the nematic side is perpendicular to the flow, is labeled "log rolling," and the right interface, where the director is oriented at $45^{\circ}$ with respect to the flow, is labeled " $45^{\circ}$." In the flow-aligned case, we average over both interfaces. The interface positions $y_{I N}$ and $y_{N I}$ themselves are used to analyze the capillary wave spectrum of the interfaces.

We should note that the two interfaces in the system are slightly correlated due to the finite width of the isotropic and the nematic slab separating them. In particular, the elastic interactions mediated through the nematic slab, which already affect the capillary wave spectrum of a single interface, ${ }^{66}$ will also cause long-range interactions between the interfaces. A detailed finite-size analysis, and data with very low statistical error, would be necessary to investigate these effects in detail. Unfortunately, this would require such an immense computational effort that it is not feasible for us at the moment. 


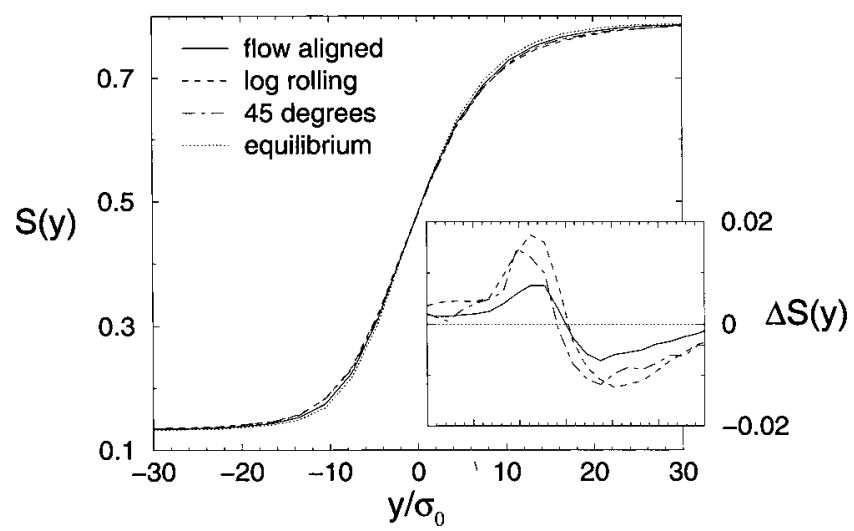

FIG. 3. Local-order-parameter profiles at average shear rate $\dot{\gamma}=0.001 / \tau$ in a system with $N=115200$ particles for the flow-aligned setup (solid), and the two interfaces from the log-rolling setup (dashed and dot dashed), compared to the corresponding equilibrium profile (dotted). The inset shows the difference $\Delta S$ between the profiles of the sheared and the equilibrium interface.

\section{RESULTS}

We first present our results for the interface at the strain rate $\dot{\gamma}=0.001 / \tau$. Some color snapshots of the system can be found in a recent preliminary report for the John-vonNeumann Institute in Jülich, which is available online. ${ }^{67}$

The stress tensor, equal to the negative pressure tensor $\mathbf{P}$, can be calculated via

$$
\boldsymbol{\sigma}=-\mathbf{P}=-\frac{1}{V}\left(\sum_{i=1}^{N} m_{i} \mathbf{v}_{i} \otimes \mathbf{v}_{i}+\sum_{i<j=1}^{N} \mathbf{r}_{i j} \otimes \mathbf{f}_{i j}\right) .
$$

The shear stress is given by $\left(\sigma_{x y}+\sigma_{y x}\right) / 2$. For the flowaligned setup, we obtained $(0.7 \pm 0.1) \times 10^{-4} \epsilon_{0} / \sigma_{0}^{3}$, and for the log-rolling setup, $(1.3 \pm 0.1) \times 10^{-4} \epsilon_{0} / \sigma_{0}^{3}$. The fact that the shear stress is higher in the log-rolling setup (i.e., the average shear viscosity is higher) is another indication that the log-rolling state is metastable. The antisymmetric stress $\sigma_{x y}-\sigma_{y x}$, as well as all other nondiagonal elements of $\boldsymbol{\sigma}$, was zero within the error. For a comparison with the equilibrium interface, we have also calculated the quantity

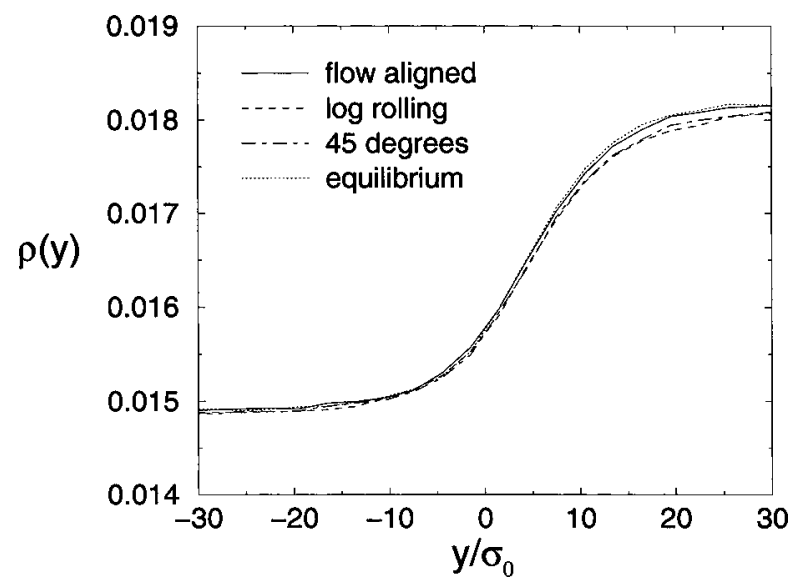

FIG. 4. Local-density profiles at $\dot{\gamma}=0.001 / \tau(N=115200$ particles $)$.

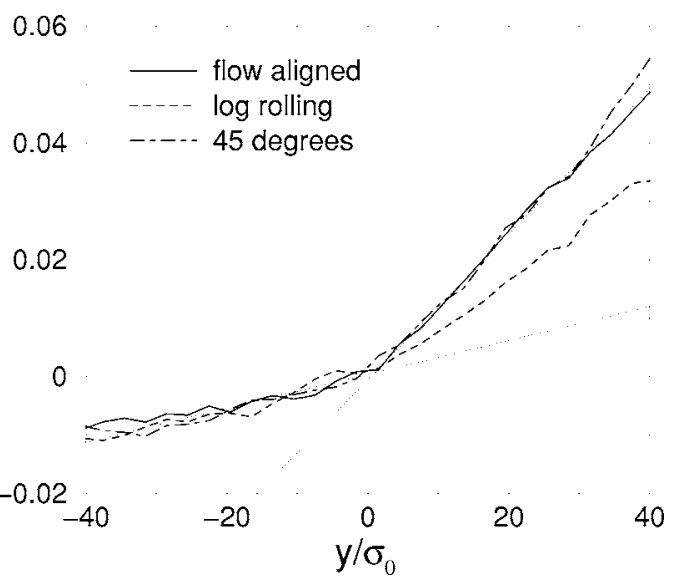

FIG. 5. Streaming velocity profiles in the flow direction, shifted such that $v_{x}=0$ at $y=0$, at $\dot{\gamma}=0.001 / \tau(N=115200$ particles $)$. The interface is located at $y=0$. The profiles from NI interfaces are mirrored at $y=0$ and $v_{x}=0$.

$$
\gamma=\frac{V}{2 A}\left(P_{y y}-\frac{1}{2}\left(P_{x x}+P_{z z}\right)\right) .
$$

In an equilibrium configuration with two interfaces in the $(x z)$ plane, $\gamma$ is the interfacial tension. The equilibrium value in our system was found to be ${ }^{42} \gamma=0.009 \pm 0.003 \epsilon_{0} \sigma_{0}$. Under shear, we obtain $\gamma=0.003 \pm 0.001 \epsilon_{0} \sigma_{0}$ in the flow-aligned setup, and $\gamma=0.015 \pm 0.001 \epsilon_{0} \sigma_{0}$ in the log-rolling setup. The difference from the equilibrium value can be attributed to two effects: First, the interfacial properties change, and second, the diagonal components of the pressure in the bulk are no longer equal under shear. On principle, these two contributions can be separated, either by systematically varying the system size in the directions parallel and perpendicular to the interface, or via a detailed analysis of the profiles of $\gamma^{41}$ Unfortunately, the first method is computationally too expensive, and the statistics of the pressure profiles was not sufficient to obtain results from the second method. Therefore, we have not been able to separate the different contributions to $\gamma$, and the interpretation of our result is difficult. The difference between the pressure tensor components $P_{x x}-P_{z z}$ is $(-3 \pm 1) \times 10^{-5} \epsilon_{0} \sigma_{0}$ in the flow-aligned setup, and zero within the error in the log-rolling setup.

The local interfacial profiles have been analyzed follow-

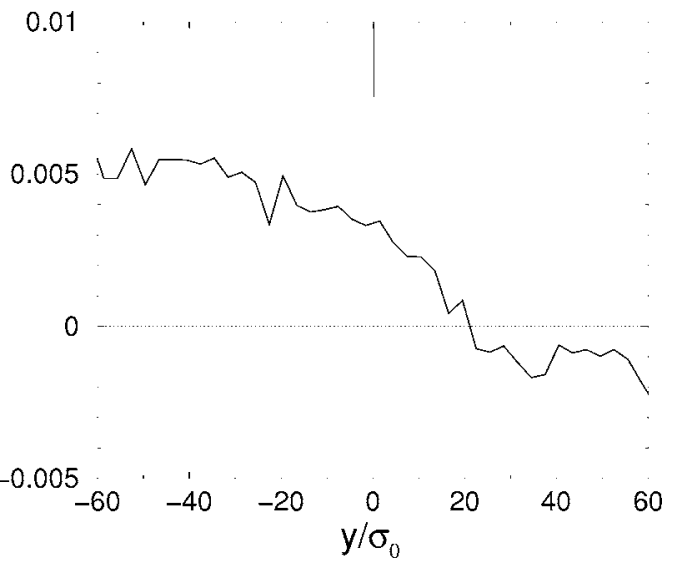

FIG. 6. Streaming velocity profile in the vorticity direction at $\dot{\gamma}=0.001 / \tau$ for the flow-aligned setup. 


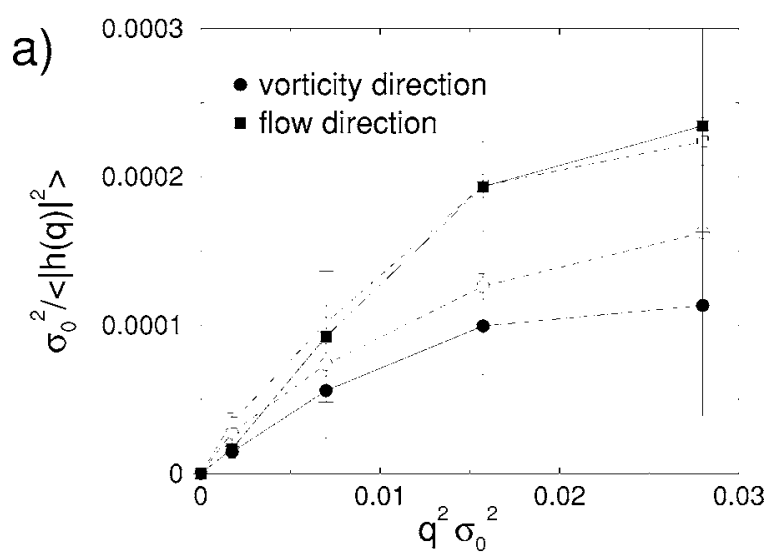

b)

0.0003
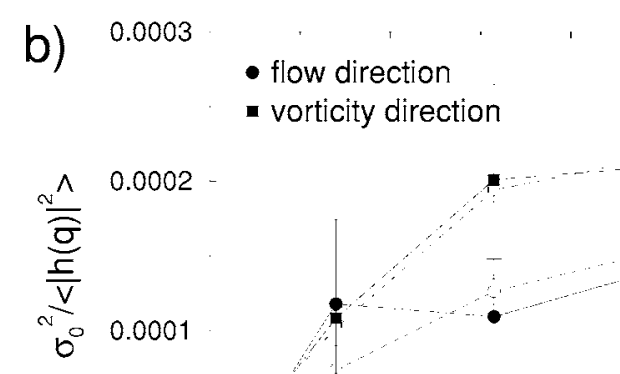

vorticity direction

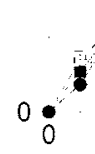

$$
\mathrm{q}^{2} \sigma_{0}{ }^{0.01}
$$

FIG. 7. Capillary wave spectrum of the interface under shear with $\dot{\gamma}$ $=0.001 / \tau(N=115200$ particles $)$ in the flow-aligned case (a) and the logrolling case (b), for wave vectors pointing in the vorticity direction and in the flow direction. The dashed lines and symbols show for comparison the equilibrium spectrum for wave vectors parallel to the director (squares, lower curve), and perpendicular to the director (circles, upper curve).

ing the procedure described in the previous section, with the lateral block size $B=37.5 \sigma_{0}$ (i.e., the system was divided into $4 \times 4=16$ columns). Figures 3 and 4 show profiles of the order parameter and the density at the interface for the flowaligned and the log-rolling setup, and compare them with the corresponding equilibrium profiles. The order-parameter profiles demonstrate that the interface broadens slightly under shear. In the log-rolling case, the effect is more pronounced than in the flow-aligned case. Moreover, the density of the coexisting nematic phase in the log-rolling case is slightly reduced.

Next we examine the streaming velocity profiles at the interface, shown in Figs. 5 and 6 . The streaming velocity profile in the flow direction clearly exhibits two different slopes: The local shear rate is smaller in the paranematic phase than in the nematic phase. Thus we observe shear banding.

Even more remarkably, the shear also induces flow in the vorticity direction $v_{z}$ (Fig. 6). The direction of that flow in the nematic and the paranematic phase is opposite. To our best knowledge, such a behavior has not been reported for homogeneous systems. (Even if it had been observed, it could be removed by a simple Galilei transformation.) Hence our effect is clearly induced by the interface. More precisely, the interface seems to induce a flow gradient in the vorticity direction. In the flow-aligned setup, where both interfaces are symmetrical, the velocity gradient dies off far from the interfaces and the nematic and the isotropic slab move as blocks in opposite directions. In the log-rolling setup, the effect is even stronger (by a factor of 3), but the situation is complicated by the fact that the two interfaces are different, and the integrated effect of the flow gradient at the two interfaces is not compatible with the periodic boundary conditions. Therefore, we only show the data for the flow-aligned case here.

In the direction of shear gradient $\left(v_{y}\right)$, no flow was observed. Likewise, the streaming angular velocity $\boldsymbol{\Omega}$ was indistinguishable from zero within the error (0.0002). In fact, $\mathbf{\Omega}$ cannot be strictly zero in the isotropic phase, since general considerations show that it should be one-half the local shear rate. ${ }^{68}$ In our system, however, the local shear rate in the isotropic slab was $\dot{\gamma} \approx 0.00025$ (cf. Fig. 6), hence the resulting $\Omega=\dot{\gamma} / 2$ is within the statistical error.

The fluctuations of the interface positions can be characterized by the capillary wave spectrum: We assume that we can parametrize the local position of an interface in the $(x, z)$ plane by a single-valued function $y=h(x, z)$. The inverse of the fluctuations of its two-dimensional Fourier transform, $\left\langle|h(\mathbf{q})|^{2}\right\rangle^{-1}$, contains information on the local interfacial free

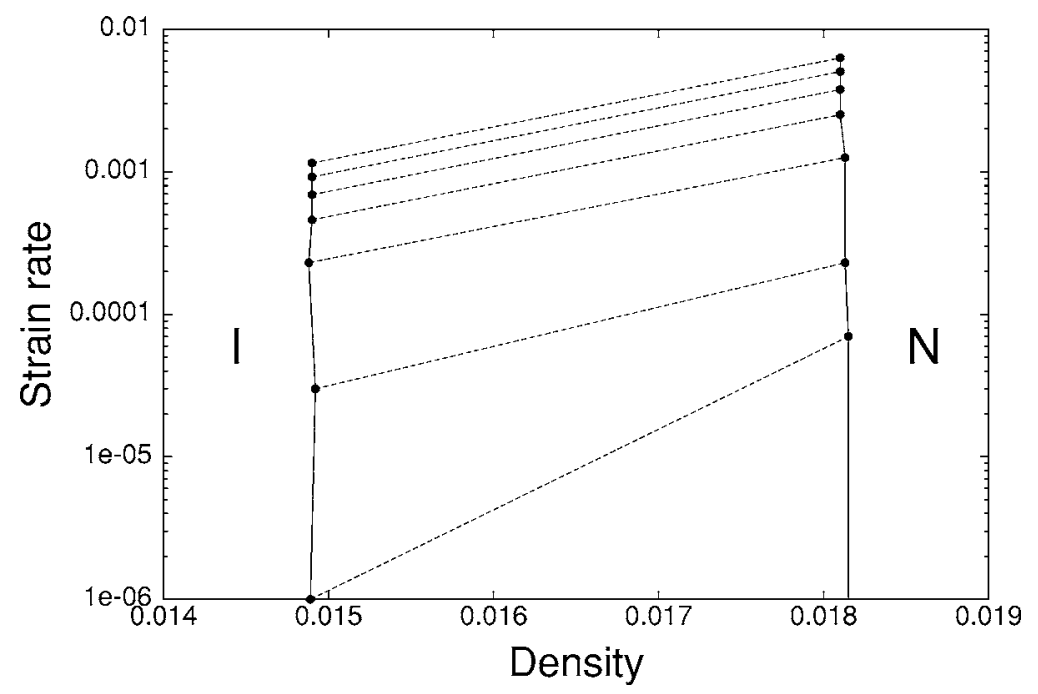

FIG. 8. Phase diagram of the flow-aligned system. The dashed "tie" lines connect coexisting states. $I$ denotes the isotropic (paranematic) region, and $N$ the nematic region. The data were collected with systems of size $N=7200$ particles. 
(a) Order parameter

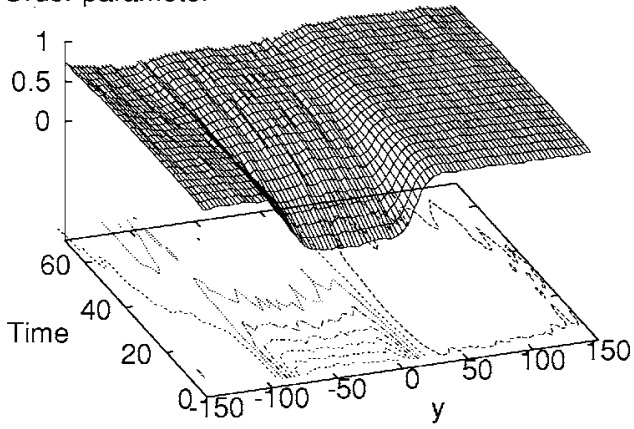

(b) Density

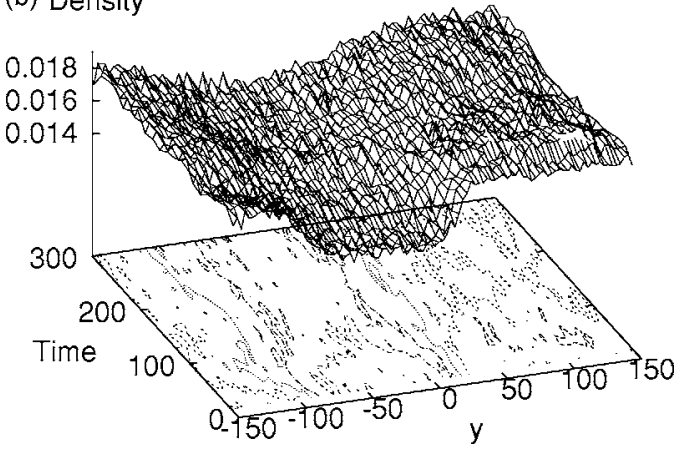

FIG. 9. Destruction of the interface in the flow-aligned system at $\dot{\gamma}=0.1 / \tau$ ( $N=115200$ particles). (a) Order-parameter profile; (b) density profile.

energy. For example, an interface whose fluctuations are solely penalized by the interfacial tension $\gamma$ has the capillary wave spectrum $^{65}$

$$
\frac{k_{B} T}{\left\langle|h(\mathbf{q})|^{2}\right\rangle}=\gamma q^{2} .
$$

The capillary waves of the equilibrium nematic/isotropic interface in our system have been investigated in detail in an earlier work. ${ }^{42}$ The spectrum was found to be anisotropic, capillary waves being stronger in the direction perpendicular to the director than in that parallel to the director. This was recently explained theoretically by Elgeti and one of us within a Landau-de Gennes treatment. ${ }^{66}$ According to the theory, the spectrum should be isotropic in the longwavelength limit $q \rightarrow 0$. However, this limit is only reached on length scales of several thousand correlation lengths. On smaller length scales, the spectrum is dominated by a strongly anisotropic cubic $q^{3}$ term and even higher-order terms.

The capillary wave spectrum is the result of a subtle interplay between the local director anchoring at the interface and the long-range elastic interactions. Since the latter are affected by shear, we expected that the spectrum should change under shear. We have performed a capillary wave analysis as described in the previous section with a lateral column size $B=18.8 \sigma_{0}$ (i.e., the system was split into $8 \times 8$ $=64$ columns). The results are shown and compared with the spectrum of the equilibrium interface in Fig. 7. Somewhat surprisingly, shear does not have much noticeable effect on the capillary wave amplitudes. At low $q$ values, corresponding to large wavelengths, the amplitudes $\left\langle|h(\mathbf{q})|^{2}\right\rangle$ are higher than those of the equilibrium interface. This indicates that

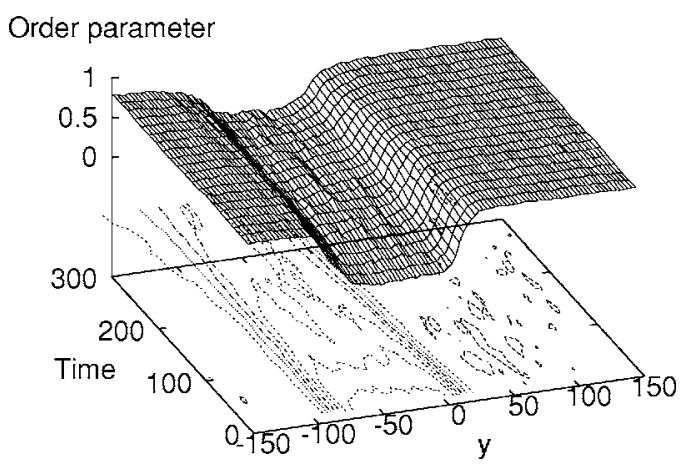

FIG. 10. Destruction of the interface in the flow-aligned system at $\dot{\gamma}$ $=0.01 / \tau(N=115200$ particles $)$.

shear might reduce the effective interface tension, and is compatible with the observation that the interfacial width broadens under shear. In the flow-aligned case, the capillary waves seem slightly enhanced in the vorticity direction. However, the effect is weak, and the deviations from the equilibrium values are still within the error bars.

Finally in this section, we discuss the phase coexistence and the stability of the interface at other strain rates. To study this, we have carried out a number of simulations of smaller, flow-aligned systems, where the length of the simulation box and the average density of the particles were the same as before, but the lateral size was reduced by a factor of 4 (7200 particles in a box with aspect ratio 1:8:1). The interfaces remain stable up to an average strain rate of roughly $\dot{\gamma}$ $=0.006 / \tau$. Figure 8 shows the corresponding nonequilibrium phase diagram. Somewhat surprisingly, the densities of the two coexisting states are almost independent of the shear rate. This underlines our earlier statement that the phase separation in our system is almost exclusively driven by thermodynamic forces. The local shear rate is always higher in the nematic phase, i.e., the system is always shear thinning upon ordering. In contrast to predictions from theoretical model calculations, ${ }^{34,35}$ the coexistence region does not close up. Rather, the interface disappears abruptly beyond the average shear rate $\dot{\gamma}=0.006 / \tau$. Coexistence regions similar to ours have been predicted for coexisting paranematic and logrolling states. ${ }^{35}$ Why we observe this structure here for paranematic states coexisting with flow-aligned nematic states is unclear, and will have to be the subject of future studies.

Figures 9 and 10 show the time evolution of interface destruction at shear rates $\dot{\gamma}=0.1 / \tau$ and $\dot{\gamma}=0.01 / \tau$. The initial configuration is an equilibrium interface. At the shear rate $\dot{\gamma}=0.1 / \tau$, the shear aligns the liquid crystal throughout the paranematic slab, thereby destroying the difference between the two phases. This is the primary process and happens on a time scale of roughly $30 \tau$. In a second step, the density slowly adjusts. The density profile therefore persists much longer than the order-parameter profile [Fig. 9(b)]. At the shear rate $\dot{\gamma}=0.01 / \tau$, the destruction process is less homogeneous and nucleates at the interfaces: They slowly move inwards and gradually lose their structure (Fig. 10). This happens on a time scale of roughly $300 \tau$. The time scale of destruction at average shear rate $0.01 / \tau$ is thus about ten 
times as large as at shear rate $0.1 / \tau$. We conclude that the time scale of destruction is roughly proportional to the time scale $1 / \dot{\gamma}$ introduced by the shear. This underlines the necessity of performing very long simulation runs at lower shear rates. In particular, it is important to note that at the shear rate $\dot{\gamma}=0.001 / \tau$, where we have performed most of our simulations, the interface showed no sign of destabilization after a run of length $6000 \tau$. Therefore we can deduce that the interface is indeed stable.

\section{CONCLUSIONS AND OUTLOOK}

We have presented an extensive nonequilibrium molecular-dynamics simulation of liquid-crystal interfaces in shear flow, and compared the properties of these interfaces with the structure of the corresponding equilibrium interface. Two situations were considered: In the first, flow-aligned setup, the interface was set up such that the director in the nematic phase pointed in the direction of shear gradient; in the second, log-rolling setup, the director pointed in the direction of the vorticity. The log-rolling state appeared to be unstable with respect to the flow-aligned state, but extremely long lived, so that it could be analyzed as well. In both the flow-aligned and the log-rolling system, the two coexisting states experience the same, common, stress. The third possible situation, phase coexistence at common strain (Fig. 1, right), was not yet studied. This will be done in future work.

We found that the structural properties of the interface are not dramatically affected by the presence of shear. The interfacial width increases slightly, and the capillary wave amplitudes are slightly enhanced at large wavelengths. No other noticeable effects were observed. For example, the biaxiality was still basically zero (data not shown).

In contrast, the streaming velocity profiles revealed a number of interesting features: First, we observe shear banding, i.e., the shear profile is inhomogeneous. The local shear rate in the nematic phase is distinctly higher than in the paranematic phase. Second and surprisingly, the interface also induces a streaming velocity gradient in the vorticity direction. As a result, vorticity flow in opposite directions is induced in the paranematic and the nematic phase. This flow is symmetry breaking, it destroys the mirror symmetry at the (xy) plane. The effect is small but significant. So far, we have no explanation. It has not been predicted by theory; however, the theoretical studies of shear banding cited in the Introduction were all set up such that the vorticity flow was zero by construction. We are not aware of any fundamental argument against nonzero vorticity flow. Hence our result is perplexing, but not impossible. We hope that it will stimulate further theoretical work on shear banding, and that the mechanism and the conditions for symmetry breaking will be clarified.

The effect is not only of academic interest, but could also be useful from a practical point of view. For example, if one component enriches in one phase, and the other in the other phase, shear could possibly be used to separate the two components.

The present study has focused on interfaces which are mainly stabilized by thermodynamic driving forces. As mentioned in the Introduction, nonequilibrium interfaces can also be stabilized mechanically. In that case, the interfacial properties presumably differ much more strongly from those of equilibrium interfaces than in the present case. We hope that we will succeed in stabilizing and studying such an interface in the future.

\section{ACKNOWLEDGMENTS}

We thank Peter Olmsted and Michael Allen for useful discussions, and Radovan Bast for running many $N=7200$ systems within an undergraduate physical chemistry project. The parallel MD program GBMEGA used in this work was originally developed by the EPSRC Complex Fluid Consortium. This work was funded by the German Science Foundation, and the simulations were carried out at the John von Neumann Institute for Computing in Jülich.

${ }^{1}$ R. G. Larson, The Structure and Rheology of Complex Fluids (Oxford University Press, New York, 1999).

${ }^{2}$ P. G. de Gennes and J. Prost, The Physics of Liquid Crystals, 2nd ed. (Oxford University Press, New York, 1993).

${ }^{3}$ A. D. Rey and M. M. Denn, Annu. Rev. Fluid Mech. 34, 233 (2002).

${ }^{4}$ M. Doi and S. F. Edwards, The Theory of Polymer Dynamics (Oxford University Press, New York, 1986).

${ }^{5}$ G. P. Morriss, P. J. Daivis, and D. J. Evans, J. Chem. Phys. 94, 7420 (1991).

${ }^{6}$ X. F. Yuan and M. P. Allen, Physica A 240, 145 (1997).

${ }^{7}$ S. Forster, M. Konrad, and P. Lindner, Phys. Rev. Lett. 94, 017803 (2005).

${ }^{8}$ J. F. Berret, S. Lerouge, and J. P. Decruppe, Langmuir 18, 7279 (2002).

${ }^{9}$ P. D. Olmsted and P. Goldbart, Phys. Rev. A 41, 4578 (1990).

${ }^{10}$ J. F. Berret, D. C. Roux, and G. Porte, J. Phys. II 4, 1261 (1994).

${ }^{11}$ J. F. Berret, D. C. Roux, G. Porte, and P. Lindner, Europhys. Lett. 25, 521 (1994).

${ }^{12}$ E. Cappelaere, J.-F. Berret, J. P. Decruppe, R. Cressely, and P. Lindner, Phys. Rev. E 56, 1869 (1997).

${ }^{13}$ J.-F. Berret, D. C. Roux, and P. Lindner, Eur. Phys. J. B 5, 67 (1998).

${ }^{14}$ P. T. Mather, A. Romo-Uribe, C. D. Han, and S. S. Kim, Macromolecules 30, 7977 (1997).

${ }^{15}$ D. Roux, F. Nallet, and O. Diat, Europhys. Lett. 24, 53 (1993).

${ }^{16}$ D. Roux, F. Nallet, and O. Diat, J. Phys. II 3, 1427 (1993).

${ }^{17}$ R. G. Larson and D. W. Mead, Liq. Cryst. 15, 151 (1993).

${ }^{18}$ J. F. Berret, D. C. Roux, G. Porte, and P. Lindner, Europhys. Lett. 32, 137 (1995).

${ }^{19}$ A. V. Zakharov, A. A. Vakulenko, and J. Thoen, J. Chem. Phys. 118, 4253 (2003)

${ }^{20}$ S. Hess and M. Kröger, J. Phys.: Condens. Matter 16, S3835 (2004).

${ }^{21}$ M. P. Lettinga and J. K. G. Dhont, J. Phys.: Condens. Matter 16, S3929 (2004).

${ }^{22}$ E. B. Bagley, I. M. Cabott, and D. C. West, J. Appl. Phys. 29, 109 (1958).

${ }^{23}$ T. C. B. McLeish and R. C. Ball, J. Polym. Sci., Polym. Phys. Ed. 24, 1735 (1986).

${ }^{24}$ M. E. Cates, T. C. B. McLeish, and G. Marrucci, Europhys. Lett. 21, 451 (1993).

${ }^{25}$ D. C. Roux, J.-F. Berret, G. Porte, E. Peuvrel-Disdier, and P. Lindner, Macromolecules 28, 1681 (1995).

${ }^{26}$ J.-F. Berret, G. Porte, and J.-P. Decruppe, Phys. Rev. E 55, 1668 (1996).

${ }^{27}$ M. M. Britton and P. T. Callaghan, Eur. Phys. J. B 7, 237 (1999).

${ }^{28}$ E. Fischer and P. T. Callaghan, Phys. Rev. E 64, 011501 (2001).

${ }^{29}$ M. R. Lopez-Gonzalez, W. M. Holmes, P. T. Callaghan, and P. J. Photinos, Phys. Rev. Lett. 93, 268302 (2004).

${ }^{30}$ P. D. Olmsted, Europhys. Lett. 48, 339 (1999).

${ }^{31}$ J. K. G. Dhont, Phys. Rev. E 60, 4534 (1999).

${ }^{32}$ P. D. Olmsted and P. Goldbart, Phys. Rev. A 46, 4966a (1992).

${ }^{33}$ G. Porte, J.-F. Berret, and J. L. Harden, J. Phys. II 7, 459 (1997)

${ }^{34}$ P. D. Olmsted and C. Y. D. Lu, Phys. Rev. E 56, R55 (1997).

${ }^{35}$ P. D. Olmsted and C. Y. D. Lu, Phys. Rev. E 60, 4397 (1999).

${ }^{36}$ V. Schmitt, C. M. Marques, and F. Lequeux, Phys. Rev. E 52, 4009 (1995).

${ }^{37}$ S. M. Fielding and P. D. Olmsted, Eur. Phys. J. E 11, 65 (2003). 
${ }^{38}$ M. A. Bates and C. Zannoni, Chem. Phys. Lett. 280, 40 (1997).

${ }^{39}$ M. P. Allen, J. Chem. Phys. 112, 5447 (2000).

${ }^{40}$ M. S. Al-Barwani and M. P. Allen, Phys. Rev. E 62, 6706 (2000).

${ }^{41}$ A. J. McDonald, M. P. Allen, and F. Schmid, Phys. Rev. E 63, 010701R (2001).

${ }^{42}$ N. Akino, F. Schmid, and M. P. Allen, Phys. Rev. E 63, 041706 (2001).

${ }^{43}$ R. L. C. Vink and T. Schilling, Phys. Rev. E 71, 051716 (2005).

${ }^{44}$ R. L. C. Vink, S. Wolfsheimer, and T. Schilling, J. Chem. Phys. 123, 074901 (2005).

${ }^{45}$ N. H. Phuong, G. Germano, and F. Schmid, J. Chem. Phys. 115, 7227 (2001).

${ }^{46}$ N. H. Phuong and F. Schmid, J. Chem. Phys. 119, 1214 (2003)

${ }^{47}$ H. Lange and F. Schmid, Eur. Phys. J. E 147, 276 (2002).

${ }^{48}$ H. Lange and F. Schmid, J. Chem. Phys. 117, 362 (2002)

${ }^{49}$ D. Baalss and S. Hess, Phys. Rev. Lett. 57, 86 (1986).

${ }^{50}$ S. Sarman and D. J. Evans, J. Chem. Phys. 99, 9021 (1993).

${ }^{51}$ S. Sarman, J. Chem. Phys. 103, 10378 (1995).

${ }^{52}$ S. Sarman, J. Chem. Phys. 107, 3144 (1997).

${ }^{53}$ S. S. Sarman, D. J. Evans, and P. T. Cummings, Phys. Rep. 305, 1 (1998).
${ }^{54}$ S. Hess, C. Aust, L. Bennett, M. Kröger, C. P. Borgmeyer, and T. Weider, Physica A 240, 126 (1997).

${ }^{55}$ L. Bennett and S. Hess, Phys. Rev. E 60, 5561 (1999).

${ }^{56}$ J. L. McWhirter and G. N. Patey, J. Chem. Phys. 117, 8551 (2002).

${ }^{57}$ J. L. McWhirter and G. N. Patey, J. Chem. Phys. 117, 2747 (2002).

${ }^{58}$ P. P. Jose and B. Bagchi, J. Chem. Phys. 121, 6978 (2004).

${ }^{59}$ B. J. Berne and P. Pechukas, J. Chem. Phys. 56, 4213 (1972).

${ }^{60}$ A. W. Lees and S. F. Edwards, J. Phys. C 5, 1921 (1972).

${ }^{61}$ M. P. Allen and D. J. Tildesley, Computer Simulation of Liquids (Oxford University Press, New York, 1989).

${ }^{62}$ D. J. Evans and G. P. Morriss, Statistical Mechanics of Nonequilibrium Fluids (Academic, San Diego, 1990).

${ }^{63}$ D. Frenkel and B. Smit, Understanding Molecular Simulation 2nd ed.,(Academic, San Diego, 2002).

${ }^{64}$ M. V. von Smoluchowski, Ann. Phys. 25, 205 (1908).

${ }^{65} \mathrm{~J}$. S. Rowlinson and B. Widom, Molecular Theory of Capillarity (Clarendon, Oxford, 1982).

${ }^{66}$ J. Elgeti and F. Schmid, Eur. Phys. J. E (submitted).

${ }^{67}$ G. Germano and F. Schmid, NIC-Series 20, 311 (2003).

${ }^{68}$ N. K. Ailawadi, B. J. Berne, and D. Forster, Phys. Rev. A 3, 1462 (1971). 